\title{
Jensen's Inequality for Generalized Peng's $g$-Expectations and Its Applications
}

\author{
Zhaojun Zong \\ School of Mathematical Sciences, Qufu Normal University, Qufu, Shandong 273165, China \\ Correspondence should be addressed to Zhaojun Zong; zongzj001@163.com
}

Received 12 November 2012; Accepted 7 January 2013

Academic Editor: Xinguang Zhang

Copyright (C) 2013 Zhaojun Zong. This is an open access article distributed under the Creative Commons Attribution License, which permits unrestricted use, distribution, and reproduction in any medium, provided the original work is properly cited.

We study Jensen's inequality for generalized Peng's $g$-expectations and give four equivalent conditions on Jensen's inequality for generalized Peng's $g$-expectations without the assumption that the generator $g$ is continuous with respect to $t$. This result includes and extends some existing results. Furthermore, we give some applications of Jensen's inequality for generalized Peng's $g$-expectations.

\section{Introduction}

By Pardoux and Peng [1], we know that there exists a unique adapted and square integrable solution to a backward stochastic differential equation (BSDE for short) of the type

$$
y_{t}=\xi+\int_{t}^{T} g\left(s, y_{s}, z_{s}\right) d s-\int_{t}^{T} z_{s} \cdot d W_{s}, \quad t \in[0, T],
$$

provided that the function $g$ is Lipschitz in both variables $y$ and $z$, and $\xi$ and $(g(t, 0,0))_{t \in[0, T]}$ are square integrable. $g$ is said to be the generator of BSDE (1). We denote the unique adapted and square integrable solution of BSDE (1) by $\left(y_{t}^{(T, g, \xi)}, z_{t}^{(T, g, \xi)}\right)_{t \in[0, T]}$.

Based on such a BSDE, Peng [2] introduced the notion of $g$-expectation. He proved that the $g$-expectation preserves many of properties of the classical mathematical expectation, but not the linearity property, and thus the $g$-expectation is a type of nonlinear mathematical expectation. Indeed, $g$ expectation is a kind of nonlinear expectation, which can be considered as a nonlinear extension of the well-known Girsanov transformations. The original motivation for studying $g$-expectation comes from the theory of expected utility. Since the notion of $g$-expectation was introduced, many properties of $g$-expectation have been investigated by many researchers. In 1997, Peng [3] introduced the notions of conditional $g$-expectation and $g$-martingale. Later, Briand et al. [4] studied Jensen's inequality for $g$-expectations and gave a counter example and a proposition to indicate that even for a linear function, Jensen's inequality might fail for some $g$-expectations. This yields a natural question: under which conditions on $g$ in the $g$-expectation does Jensen's inequality hold for any convex function? Under the assumptions that $g$ does not depend on $y$ and is convex, Chen et al. $[5,6]$ studied Jensen's inequality for $g$-expectations and gave a necessary and sufficient condition on $g$ under which Jensen's inequality holds for convex functions. Provided that $g$ only does not depend on $y$, Jiang [7] gave another necessary and sufficient condition on $g$ under which Jensen's inequality holds for convex functions. It was an improved result in comparison with the result that Chen et al. yielded. Later, this result was improved by $\mathrm{Hu}$ [8] and Jiang [9] showing that, in fact, $g$ must be independent of $y$. But these results need the assumption that the generator $g$ is continuous with respect to $t$.

In this paper, without the assumption that the generator $g$ is continuous with respect to $t$, we study Jensen's inequality for generalized Peng's $g$-expectations and give four equivalent conditions on Jensen's inequality for generalized Peng's $g$ expectations, which generalize the known results on Jensen's inequality for $g$-expectations in Chen et al. [5, 6], Jiang [7, 9], and $\mathrm{Hu}$ [8]. Furthermore, we give some applications of Jensen's inequality for generalized Peng's $g$-expectations.

This paper is organized as follows: in Section 2, we introduce some notations, assumptions, notions, and lemmas 
which will be useful in this paper; in Section 3, we give our main results including the proofs and applications.

\section{Preliminaries}

Firstly, let us list some notations, assumptions, notions, lemmas, and propositions that are used in this paper. Let $(\Omega, \mathscr{F}, P)$ be a probability space and let $\left(W_{t}\right)_{t \geq 0}$ be a $d$ dimensional standard Brownian motion with respect to filtration $\left(\mathscr{F}_{t}\right)_{t \geq 0}$ generated by Brownian motion and all $P$ null subsets, that is,

$$
\mathscr{F}_{t}=\sigma\left\{W_{s} ; s \leq t\right\} \vee \mathcal{N},
$$

where $\mathcal{N}$ is the set of all $P$-null subsets. Fix a real number $T>0$. For any positive integer $n$ and $z \in R^{n},|z|$ denotes its Euclidean norm.

We define the following usual spaces of processes (random variables):

(i) Consider $L^{p}\left(\Omega, \mathscr{F}_{T}, P\right)=\left\{\xi: \xi\right.$ is $\mathscr{F}_{T}$-measurable random variable such that $\left.E\left[|\xi|^{p}\right]<\infty, p \geq 1\right\}$;

(ii) Consider $\mathscr{L}\left(\Omega, \mathscr{F}_{T}, P\right)=\bigcup_{p>1} L^{p}\left(\Omega, \mathscr{F}_{T}, P\right)$;

(iii) Consider $\mathcal{S}_{\mathscr{F}}^{P}(0, T ; P ; R)=\{V: V$ is a continuous process with $\left.E\left[\sup _{0 \leq t \leq T}\left|V_{t}\right|^{p}\right]<\infty, p \geq 1\right\}$;

(iv) Consider $\mathcal{S}_{\mathscr{F}}(0, T ; P ; R)=\bigcup_{p>1} \mathcal{S}_{\mathscr{F}}^{p}(0, T ; P ; R)$;

(v) Consider $\mathscr{L}_{\mathscr{F}}^{p}\left(0, T ; P ; R^{n}\right)=\{V: V$ is a progressively measurable process with $E\left[\left(\int_{0}^{T}\left|V_{s}\right|^{2} d s\right)^{p / 2}\right]<$ $\infty, p \geq 1\} ;$

(vi) Consider $\mathscr{L}_{\mathscr{F}}\left(0, T ; P ; R^{n}\right)=\bigcup_{p>1} L_{\mathscr{F}}^{p}\left(0, T ; P ; R^{n}\right)$.

Suppose the generator $g(\omega, t, y, z): \Omega \times[0, T] \times R \times R^{d} \mapsto$ $R$ satisfies the following assumptions:

(A.1) there exists a constant $\mu>0$, such that $P$-a.s., we have: $\forall t \in[0, T], \forall y_{1}, y_{2} \in R, z_{1}, z_{2} \in R^{d}, \mid g\left(t, y_{1}, z_{1}\right)-$ $g\left(t, y_{2}, z_{2}\right) \mid \leq \mu\left(\left|y_{1}-y_{2}\right|+\left|z_{1}-z_{2}\right|\right)$;

(A.2) $P$-a.s., $\forall(t, y) \in[0, T] \times R, g(t, y, 0) \equiv 0$.

The following lemma is a special case of Theorem 4.2 in Briand et al. [10].

Lemma 1. Suppose g satisfies (A.1) and (A.2). Then for each given $\xi \in L^{p}\left(\Omega, \mathscr{F}_{T}, P\right)$, where $1<p<2$, the BSDE (1) has a unique pair of adapted processes $\left(y_{t}^{(T, g, \xi)}, z_{t}^{(T, g, \xi)}\right)_{t \in[0, T]} \in$ $\mathcal{S}_{\mathscr{F}}^{p}(0, T ; P ; R) \times l_{\mathscr{F}}^{p}\left(0, T ; P ; R^{d}\right)$.

From Lemma 1, we have the following.

Remark 2. Suppose $g$ satisfies (A.1) and (A.2). Then for each given $\xi \in \mathscr{L}\left(\Omega, \mathscr{F}_{T}, P\right)$, the BSDE (1) has a unique pair of adapted processes $\left(y_{t}^{(T, g, \xi)}, z_{t}^{(T, g, \xi)}\right)_{t \in[0, T]} \in \mathcal{S}_{\mathscr{F}}(0, T ; P ; R) \times$ $\mathscr{L}_{\mathscr{F}}\left(0, T ; P ; R^{d}\right)$.

Now, we introduce the notions of generalized Peng's $g$-expectation and generalized conditional Peng's $g$ expectation.
Definition 3 (generalized Peng's $g$-expectation [11]). Suppose $g$ satisfies (A.1) and (A.2). For any $\xi \in \mathscr{L}\left(\Omega, \mathscr{F}_{T}, P\right)$, let $\left(y_{t}^{(T, g, \xi)}, z_{t}^{(T, g, \xi)}\right)_{t \in[0, T]}$ be the solution of BSDE (1). Consider the mapping $\mathscr{E}_{g}[\cdot]: \mathscr{L}\left(\Omega, \mathscr{F}_{T}, P\right) \mapsto R$, denoted by $\mathscr{E}_{g}[\xi]=$ $y_{0}^{(T, g, \xi)}$. One calls $\mathscr{E}_{g}[\xi]$ the generalized Peng's $g$-expectation of $\xi$.

Definition 4 (generalized Peng's conditional $g$-expectation [11]). Suppose $g$ satisfies (A.1) and (A.2). The generalized Peng's conditional $g$-expectation of $\xi$ with respect to $\mathscr{F}_{t}$ is defined by

$$
\mathscr{E}_{g}\left[\xi \mid \mathscr{F}_{t}\right]=y_{t}^{(T, g, \xi)}, \quad t \in[0, T]
$$

Then, let us list some basic properties of generalized Peng's $g$-expectation.

Proposition 5 (see [11]). Consider $\mathscr{E}_{g}\left[\xi \mid \mathscr{F}_{t}\right]$ is the unique random variable $\eta$ in $\mathscr{L}\left(\Omega, \mathscr{F}_{t}, P\right)$ such that

$$
\mathscr{E}_{g}\left[1_{A} \xi\right]=\mathscr{E}_{g}\left[1_{A} \eta\right], \quad \forall A \in \mathscr{F}_{t}
$$

Proposition 6 (see [11]). Suppose g satisfies (A.1) and (A.2). If $g$ does not depend on $y$, that is, $g(\omega, t, z): \Omega \times[0, T] \times R^{d} \mapsto R$, then

$$
\begin{aligned}
\mathscr{E}_{g}\left[\xi+\eta \mid \mathscr{F}_{t}\right]=\mathscr{E}_{g}\left[\xi \mid \mathscr{F}_{t}\right]+\eta, \quad & \forall \xi \in \mathscr{L}\left(\Omega, \mathscr{F}_{T}, P\right), \\
& \forall \eta \in \mathscr{L}\left(\Omega, \mathscr{F}_{t}, P\right) .
\end{aligned}
$$

Proposition 7 (see [11]). Suppose g satisfies (A.1) and (A.2). For $\xi, \eta_{n} \in L^{p}\left(\Omega, \mathscr{F}_{T}, P\right)$, where $n=1,2, \ldots$ and $p>1$, if $E\left[\left|\xi-\eta_{n}\right|^{p} \mid \mathscr{F}_{t}\right] \rightarrow 0$, a.s., $t \in[0, T]$, then

$$
\lim _{n \rightarrow \infty} \mathscr{E}_{g}\left[\eta_{n} \mid \mathscr{F}_{t}\right]=\mathscr{E}_{g}\left[\xi \mid \mathscr{F}_{t}\right], \quad \text { a.s. }, t \in[0, T]
$$

Applying Proposition 7, one can immediately obtain the following.

Remark 8. (i) For any $\xi \in \mathscr{L}\left(\Omega, \mathscr{F}_{T}, P\right)$, let $\xi^{n}=(\xi \wedge n) \vee(-n)$, $n=1,2, \ldots$, then $\lim _{n \rightarrow \infty} \mathscr{E}_{g}\left[\xi^{n} \mid \mathscr{F}_{t}\right]=\mathscr{E}_{g}\left[\xi \mid \mathscr{F}_{t}\right]$, a.s., $\forall t \in[0, T]$.

(ii) For any $\xi_{n} \in \mathscr{L}\left(\Omega, \mathscr{F}_{T}, P\right)$, if $\lim _{n \rightarrow \infty} \xi_{n}=\xi$ a.s. and $\left|\xi_{n}\right| \leq \eta$ a.s. with $\eta \in \mathscr{L}\left(\Omega, \mathscr{F}_{T}, P\right)$, then $\lim _{n \rightarrow \infty} \mathscr{E}_{g}\left[\xi^{n} \mid\right.$ $\left.\mathscr{F}_{t}\right]=\mathscr{E}_{g}\left[\xi \mid \mathscr{F}_{t}\right]$, a.s., $\forall t \in[0, T]$.

Lemma 9. Suppose $g$ satisfies (A.1) and (A.2). Let $\left\{A_{i}\right\}_{i=1}^{m}$ be a $\mathscr{F}_{t}$-measurable partition of $\Omega$ (i.e., $A_{i} \in \mathscr{F}_{t}, A_{i} \cap A_{j}=\emptyset$ if $i \neq j$ and $\bigcup_{i=1}^{m} A_{i}=\Omega$ ), where $t \leq T$. Then for each $X_{i} \in$ $\mathscr{L}\left(\Omega, \mathscr{F}_{T}, P\right), i=1, \ldots, m$, one has

$$
\sum_{i=1}^{m} 1_{A_{i}} \mathscr{E}_{g}\left[X_{i} \mid \mathscr{F}_{t}\right]=\mathscr{E}_{g}\left[\sum_{i=1}^{m} 1_{A_{i}} X_{i} \mid \mathscr{F}_{t}\right] \text { a.s. }
$$


Proof. We consider the following BSDEs:

$$
\begin{aligned}
\mathscr{E}_{g} & {\left[X_{i} \mid \mathscr{F}_{t}\right] } \\
= & X_{i}+\int_{t}^{T} g\left(s, \mathscr{E}_{g}\left[X_{i} \mid \mathscr{F}_{s}\right], z_{s}^{\left(T, g, X_{i}\right)}\right) d s \\
& \quad-\int_{t}^{T} z_{s}^{\left(T, g, X_{i}\right)} \cdot d W_{s}, \quad i=1, \ldots, m, \\
\mathscr{E}_{g}\left[\sum_{i=1}^{m} 1_{A_{i}} X_{i} \mid \mathscr{F}_{t}\right] & \sum_{i=1}^{m} 1_{A_{i}} X_{i}+\int_{t}^{T} g\left(s, \mathscr{E}_{g}\left[\sum_{i=1}^{m} 1_{A_{i}} X_{i} \mid \mathscr{F}_{s}\right],\right. \\
& -\int_{t}^{T} z_{s}^{\left(T, g, \sum_{i=1}^{m} 1_{A_{i}} X_{i}\right)} \cdot d W_{s} .
\end{aligned}
$$

By the fact that $\sum_{i=1}^{m} 1_{A_{i}} g\left(s, \mathscr{E}_{g}\left[X_{i} \mid \mathscr{F}_{s}\right], z_{s}^{\left(T, g, X_{i}\right)}\right)=$ $g\left(s, \sum_{i=1}^{m} 1_{A_{i}} \mathscr{E}_{g}\left[X_{i} \mid \mathscr{F}_{s}\right], \sum_{i=1}^{m} 1_{A_{i}} z_{s}^{\left(T, g, X_{i}\right)}\right), t \leq s \leq T$ and from (8), we have

$$
\begin{aligned}
\sum_{i=1}^{m} 1_{A_{i}} \mathscr{E}_{g}\left[X_{i} \mid \mathscr{F}_{t}\right] & \\
= & \sum_{i=1}^{m} 1_{A_{i}} X_{i} \\
& +\int_{t}^{T} g\left(s, \sum_{i=1}^{m} 1_{A_{i}} \mathscr{E}_{g}\left[X_{i} \mid \mathscr{F}_{s}\right], \sum_{i=1}^{m} 1_{A_{i}} z_{s}^{\left(T, g, X_{i}\right)}\right) d s \\
& -\int_{t}^{T} \sum_{i=1}^{m} 1_{A_{i}} z_{s}^{\left(T, g, X_{i}\right)} \cdot d W_{s} .
\end{aligned}
$$

Comparing this with (9), it follows that $\sum_{i=1}^{m} 1_{A_{i}} \mathscr{E}_{g}\left[X_{i}\right.$ $\left.\mathscr{F}_{t}\right]=\mathscr{E}_{g}\left[\sum_{i=1}^{m} 1_{A_{i}} X_{i} \mid \mathscr{F}_{t}\right]$ a.s. The proof of Lemma 9 is complete.

Proposition 10. Suppose g satisfies (A.1) and (A.2). Then the following two statements are equivalent:

(i) consider $\forall(X, k) \in \mathscr{L}\left(\Omega, \mathscr{F}_{T}, P\right) \times R, \mathscr{E}_{g}\left[X+k \mid \mathscr{F}_{t}\right]=$ $\mathscr{E}_{g}\left[X \mid \mathscr{F}_{t}\right]+k$ a.s.,

(ii) consider $\forall(X, \eta) \in \mathscr{L}\left(\Omega, \mathscr{F}_{T}, P\right) \times \mathscr{L}\left(\Omega, \mathscr{F}_{t}, P\right)$, $\mathscr{E}_{g}\left[X+\eta \mid \mathscr{F}_{t}\right]=\mathscr{E}_{g}\left[X \mid \mathscr{F}_{t}\right]+\eta$ a.s.

Proof. It is obvious that (ii) implies (i). We only need to prove that (i) implies (ii). Suppose (i) holds. Let $\left\{A_{i}\right\}_{i=1}^{m}$ be a $\mathscr{F}_{t}$-measurable partition of $\Omega$ and let $\lambda_{i} \in R(i=$
$1,2, \ldots, m)$. From Lemma 9 and (i), we deduce that for each $X \in \mathscr{L}\left(\Omega, \mathscr{F}_{T}, P\right)$,

$$
\begin{aligned}
\mathscr{E}_{g}\left[X+\sum_{i=1}^{m} \lambda_{i} 1_{A_{i}} \mid \mathscr{F}_{t}\right] & =\mathscr{E}_{g}\left[\sum_{i=1}^{m} 1_{A_{i}}\left(X+\lambda_{i}\right) \mid \mathscr{F}_{t}\right] \\
& =\sum_{i=1}^{m} 1_{A_{i}} \mathscr{E}_{g}\left[X+\lambda_{i} \mid \mathscr{F}_{t}\right] \\
& =\sum_{i=1}^{m} 1_{A_{i}}\left(\mathscr{E}_{g}\left[X \mid \mathscr{F}_{t}\right]+\lambda_{i}\right) \\
& =\mathscr{E}_{g}\left[X \mid \mathscr{F}_{t}\right]+\sum_{i=1}^{m} \lambda_{i} 1_{A_{i}} \text { a.s. }
\end{aligned}
$$

In other words, for any $X \in \mathscr{L}\left(\Omega, \mathscr{F}_{T}, P\right)$ and any simple function $\eta \in \mathscr{L}\left(\Omega, \mathscr{F}_{t}, P\right)$,

$$
\mathscr{E}_{g}\left[X+\eta \mid \mathscr{F}_{t}\right]=\mathscr{E}_{g}\left[X \mid \mathscr{F}_{t}\right]+\eta \quad \text { a.s. }
$$

Let

$$
\begin{aligned}
\eta_{n}:= & \sum_{i=0}^{n 2^{n}-1} \frac{i}{2^{n}} 1_{\left\{\left(i / 2^{n}\right) \leq \eta<\left((i+1) / 2^{n}\right)\right\}}+n 1_{\{\eta \geq n\}} \\
& +\sum_{i=0}^{n 2^{n}-1} \frac{-i}{2^{n}} 1_{\left\{-\left((i+1) / 2^{n}\right) \leq \eta<-\left(i / 2^{n}\right)\right\}} \\
& +(-n) 1_{\{\eta<-n\}}, \quad n=1,2, \ldots .
\end{aligned}
$$

Obviously, for each $n, \eta_{n}$ is a simple function in $\mathscr{L}\left(\Omega, \mathscr{F}_{t}, P\right)$. From (12), we have

$$
\mathscr{E}_{g}\left[X+\eta_{n} \mid \mathscr{F}_{t}\right]=\mathscr{E}_{g}\left[X \mid \mathscr{F}_{t}\right]+\eta_{n} \text { a.s. }
$$

On the other hand, $\lim _{n \rightarrow \infty}\left(X+\eta_{n}\right)=X+\eta,\left|X+\eta_{n}\right| \leq$ $|X|+|\eta|$. Thus, from Remark 8 (ii), it follows that (ii) is true. The proof of Proposition 10 is complete.

\section{Main Results and Applications}

Definition 11. Let $g: \Omega \times[0, T] \times R \times R^{d} \mapsto R$. The function $g$ is said to be superhomogeneous if for each $(y, z) \in R \times R^{d}$ and any real number $\lambda$, then $g(t, \lambda y, \lambda z) \geq \lambda g(t, y, z), d P \times d t$ a.s. The function $g$ is said to be positively homogeneous if for each $(y, z) \in R \times R^{d}$ and any real number $\lambda \geq 0$, then $g(t, \lambda y, \lambda z)=\lambda g(t, y, z), d P \times d t$ a.s.

Before we give our main results, let us see an example.

Example 12. Fix $T=1$ and $d=1$. Let $\xi=f\left(W_{1}\right)$, where $f(x)=\exp \left(\left(x^{2} / 2 p_{1}\right)-x\right) 1_{\left(x \geq p_{1}\right)}, 1<p_{1}<2$.

Obviously, $f$ is an increasing function. We can easily get

$$
\begin{aligned}
E\left[|\xi|^{p_{1}}\right] & =\int_{p_{1}}^{\infty} \exp \left(\frac{x^{2}}{2}-p_{1} x\right) \frac{1}{\sqrt{2 \pi}} \mathrm{e}^{-(1 / 2) x^{2}} d x \\
& =\frac{1}{\sqrt{2 \pi} p_{1}} \mathrm{e}^{-p_{1}^{2}}<\infty, \quad E\left[|\xi|^{p}\right]=\infty, \quad \forall p>p_{1} .
\end{aligned}
$$

Hence, $\xi \in \mathscr{L}\left(\Omega, \mathscr{F}_{1}, P\right)$, but $\xi \notin L^{2}\left(\Omega, \mathscr{F}_{1}, P\right)$. 
Let $\xi^{n}=\xi \wedge n, n=1,2, \ldots$ Clearly, for each $n, \xi^{n} \in$ $L^{2}(\Omega, \mathscr{F}, P)$. For simplicity, we will write $\mathscr{E}^{\mu}[\cdot] \equiv \mathscr{E}_{g}[\cdot]$ for $g=\mu|z|$. From Theorem 1 in Chen and Kulperger's [12], we know that $\mathscr{E}^{\mu}\left[\xi^{n}\right]=E_{\mathrm{Q}}\left[\xi^{n}\right]$, where $d Q / d P=\mathrm{e}^{-(1 / 2) \mu^{2}+\mu W_{1}}$.

By Remark 8(i), we have $\mathscr{E}^{\mu}\left[\xi^{n}\right] \rightarrow \mathscr{E}^{\mu}[\xi]$, as $n \rightarrow \infty$. On the other hand, applying Hölder's inequality and noting that $E\left[\mathrm{e}^{-(1 / 2) \mu^{2}+\mu W_{1}}\right]=1$ and $E\left[\mathrm{e}^{-(1 / 2) \mu^{2} q^{2}+\mu q W_{1}}\right]=1$, we obtain

$$
\begin{aligned}
E_{\mathrm{Q}}[\xi] & \leq\left(E\left[|\xi|^{p_{1}}\right]\right)^{1 / p_{1}}\left(E\left[\left(\frac{d Q}{d P}\right)^{q}\right]\right)^{1 / q} \\
& \leq \mathrm{e}^{(1 / 2)(q-1) \mu^{2}}\left(E\left[|\xi|^{p_{1}}\right]\right)^{1 / p_{1}}<\infty,
\end{aligned}
$$

where $\left(1 / p_{1}\right)+(1 / q)=1$. It then follows from the monotonic convergence theorem that

$$
E_{\mathrm{Q}}\left[\xi^{n}\right] \longrightarrow E_{\mathrm{Q}}[\xi], \quad \text { as } n \longrightarrow \infty .
$$

Thus

$$
\mathscr{E}^{\mu}[\xi]=E_{\mathrm{Q}}[\xi] .
$$

Let $\varphi(x)=(x-k)^{+}$, where $k \in R$. Obviously, $\varphi(x)$ is a convex and increasing function. From this, we know that $\varphi \circ f$ is an increasing function. In a similar manner of the above, we can deduce that

$$
\mathscr{E}^{\mu}[\varphi(\xi)]=E_{Q}[\varphi(\xi)] .
$$
have

From (18), (19), and the classical Jensen's inequality, we

$$
\varphi\left(\mathscr{E}^{\mu}[\xi]\right)=\varphi\left(E_{\mathrm{Q}}[\xi]\right) \leq E_{\mathrm{Q}}[\varphi(\xi)]=\mathscr{E}^{\mu}[\varphi(\xi)] .
$$

This problem yields a natural question: in general, under which conditions on $g$ do generalized Peng's $g$-expectations satisfy Jensen's inequality for convex functions?

The following theorem will answer this question.

Theorem 13. Let $g$ satisfy (A.1) and (A.2). Then the following four statements are equivalent.

(i) Jensen's inequality for generalized Peng's g-expectation $\mathscr{E}_{g}\left[\cdot \mid \mathscr{F}_{t}\right]$ holds in general, that is, for each convex function $\varphi(x): R \mapsto R$ and each $\xi \in \mathscr{L}\left(\Omega, \mathscr{F}_{T}, P\right)$, if $\varphi(\xi) \in \mathscr{L}\left(\Omega, \mathscr{F}_{T}, P\right)$, then one has

$$
\mathscr{E}_{g}\left[\varphi(\xi) \mathscr{F}_{t}\right] \geq \varphi\left(\mathscr{E}_{g}\left[\xi \mid \mathscr{F}_{t}\right]\right) \quad \text { a.s.; }
$$

(ii) consider $\forall(\xi, a, b) \in L^{2}\left(\Omega, \mathscr{F}_{T}, P\right) \times R \times R, \mathscr{E}_{g}[a \xi+$ $b] \geq a \mathscr{E}_{g}[\xi]+b$;

(iii) consider $\forall(\xi, a, b) \in L^{2}\left(\Omega, \mathscr{F}_{T}, P\right) \times R \times R, \mathscr{E}_{g}[a \xi+b \mid$ $\left.\mathscr{F}_{t}\right] \geq a \mathscr{E}_{g}\left[\xi \mid \mathscr{F}_{t}\right]+b$ a.s.;

(iv) consider $g$ is independent of $y$, superhomogeneous, and positively homogeneous with respect to $z$.
Proof. (i) $\Rightarrow$ (ii) is obvious.

(ii) $\Rightarrow$ (iii): let $\eta=\xi+b$. By (ii), we have

$$
\mathscr{E}_{g}[\eta-b] \geq \mathscr{E}_{g}[\eta]-b \text {, }
$$

That is,

$$
\mathscr{E}_{g}[\xi]+b \geq \mathscr{E}_{g}[\xi+b] .
$$

Thus, for each $(\xi, b) \in L^{2}\left(\Omega, \mathscr{F}_{T}, P\right) \times R$,

$$
\mathscr{E}_{g}[\xi+b]=\mathscr{E}_{g}[\xi]+b .
$$

For each $(X, t, k) \in L^{2}\left(\Omega, \mathscr{F}_{T}, P\right) \times[0, T] \times R$, by (24), we know that for each $A \in \mathscr{F}_{t}$,

$$
\begin{aligned}
\mathscr{E}_{g}\left[1_{A}(X+k)\right] & =\mathscr{E}_{g}\left[1_{A} X+1_{A} k-k\right]+k \\
& =\mathscr{E}_{g}\left[1_{A} X+1_{A^{C}}(-k)\right]+k \\
& =\mathscr{E}_{g}\left[\mathscr{E}_{g}\left[1_{A} X+1_{A^{C}}(-k) \mid \mathscr{F}_{t}\right]\right]+k \\
& =\mathscr{E}_{g}\left[1_{A} \mathscr{E}_{g}\left[X \mid \mathscr{F}_{t}\right]+1_{A^{C}}(-k)\right]+k \\
& =\mathscr{E}_{g}\left[1_{A} \mathscr{E}_{g}\left[X \mid \mathscr{F}_{t}\right]+1_{A^{C}}(-k)+k\right] \\
& =\mathscr{E}_{g}\left[1_{A}\left(\mathscr{E}_{g}\left[X \mid \mathscr{F}_{t}\right]+k\right)\right] .
\end{aligned}
$$

Thus,

$$
\mathscr{E}_{g}\left[X+k \mid \mathscr{F}_{t}\right]=\mathscr{E}_{g}\left[X \mid \mathscr{F}_{t}\right]+k \text { a.s., } \forall t \in[0, T] .
$$

On the other hand, for each $\lambda \neq 0$, define

$$
\mathscr{E}^{\lambda}\left[\cdot \mid \mathscr{F}_{t}\right]=\frac{\mathscr{E}_{g}\left[\lambda \cdot \mid \mathscr{F}_{t}\right]}{\lambda}, \quad \forall t \in[0, T] .
$$

It is easy to check that $\mathscr{E}_{g}\left[\cdot \mid \mathscr{F}_{t}\right]$ and $\mathscr{E}^{\lambda}\left[\cdot \mid \mathscr{F}_{t}\right]$ are two $\mathscr{F}$ expectations on $L^{2}\left(\Omega, \mathscr{F}_{T}, P\right.$ ) (the notion of $\mathscr{F}_{\text {-expectation }}$ can be seen in [13]). From (ii), we have if $\lambda>0$, for each $\xi \in L^{2}\left(\Omega, \mathscr{F}_{T}, P\right)$

$$
\mathscr{E}^{\lambda}[\xi] \geq \mathscr{E}_{g}[\xi] .
$$

Hence, by Lemma 4.5 in [13], we have

$$
\mathscr{E}^{\lambda}\left[\xi \mid \mathscr{F}_{t}\right] \geq \mathscr{E}_{g}\left[\xi \mid \mathscr{F}_{t}\right] \quad \text { a.s., } \forall t \in[0, T] .
$$

Similarly, if $\lambda<0$, for each $\xi \in L^{2}\left(\Omega, \mathscr{F}_{T}, P\right)$

$$
\mathscr{E}^{\lambda}[\xi] \leq \mathscr{E}_{g}[\xi] .
$$

Hence, by Lemma 4.5 in [13] again, we have

$$
\mathscr{E}^{\lambda}\left[\xi \mid \mathscr{F}_{t}\right] \leq \mathscr{E}_{g}\left[\xi \mid \mathscr{F}_{t}\right] \quad \text { a.s., } \forall t \in[0, T] .
$$

Thus from (29) and (31), we have $\forall(\xi, \lambda) \in L^{2}\left(\Omega, \mathscr{F}_{T}, P\right) \times R$,

$$
\mathscr{E}_{g}\left[\lambda \xi \mid \mathscr{F}_{t}\right] \geq \lambda \mathscr{E}_{g}\left[\xi \mid \mathscr{F}_{t}\right] \quad \text { a.s., } \forall t \in[0, T] .
$$


From (26) and (32), we have

$$
\begin{array}{r}
\forall(\xi, a, b) \in L^{2}\left(\Omega, \mathscr{F}_{T}, P\right) \times R \times R, \\
\mathscr{E}_{g}\left[a \xi+b \mid \mathscr{F}_{t}\right] \geq a \mathscr{E}_{g}\left[\xi \mid \mathscr{F}_{t}\right]+b \text { a.s., } \\
\forall t \in[0, T] .
\end{array}
$$

(iii) $\Rightarrow$ (iv): Firstly, we prove that $g$ is independent of $y$. From (iii), we can obtain that for each $(\xi, y) \in L^{2}\left(\Omega, \mathscr{F}_{T}, P\right) \times$ $R$,

$$
\mathscr{E}_{g}\left[\xi-y \mid \mathscr{F}_{t}\right]=\mathscr{E}_{g}\left[\xi \mid \mathscr{F}_{t}\right]-y, \quad \text { a.s., } \forall t \in[0, T] .
$$

For each $(t, y, z) \in[0, T] \times R \times R^{d}$, let $Y^{t, y, z}$ be the solution of the following SDE defined on $[t, T]$ :

$$
Y_{s}^{t, y, z}=y-\int_{t}^{s} g\left(r, Y_{r}^{t, y, z}, z\right) d r+z \cdot\left(W_{s}-W_{t}\right) .
$$

From (34), we have

$$
\begin{array}{r}
Y_{r}^{t, y, z}-y=\mathscr{E}_{g}\left[Y_{s}^{t, y, z} \mid \mathscr{F}_{r}\right]-y=\mathscr{E}_{g}\left[Y_{s}^{t, y, z}-y \mid \mathscr{F}_{r}\right], \\
t \leq r \leq s \leq T .
\end{array}
$$

Let $Y_{s}=Y_{s}^{t, y, z}-y, s \in[t, T]$ and $Z$ be the corresponding part of Itô's integrand. It then follows that

$$
\begin{aligned}
Y_{s} & =-\int_{t}^{s} g\left(r, Y_{r}^{t, y, z}, z\right) d r+\int_{t}^{s} z \cdot d W_{r} \\
& =-\int_{t}^{s} g\left(r, Y_{r}, Z_{r}\right) \mathrm{d} r+\int_{t}^{s} Z_{r} \cdot d W_{r} .
\end{aligned}
$$

Thus, $Z_{r} \equiv z$ and

$$
g\left(r, Y_{r}, z\right)=g\left(r, Y_{r}^{t, y, z}-y, z\right)=g\left(r, Y_{r}^{t, y, z}, z\right) .
$$

Then, we can apply Lemma 4.4 in Peng [14] to obtain that for each $(y, z) \in R \times R^{d}$,

$$
g(t, y, z)=g(t, 0, z), \quad d P \times d t \text { a.s. }
$$

Namely, $g$ is independent of $y$.

Now we prove that $g$ is superhomogeneous with respect to $z$. From (iii), we can obtain that for each $(\xi, \lambda) \in$ $L^{2}\left(\Omega, \mathscr{F}_{T}, P\right) \times R$,

$$
\lambda \mathscr{E}_{g}\left[\xi \mid \mathscr{F}_{t}\right] \leq \mathscr{E}_{g}\left[\lambda \xi \mid \mathscr{F}_{t}\right], \quad \text { a.s., } \forall t \in[0, T] .
$$

For each $(t, z) \in[0, T] \times R^{d}$, let $Y^{t, z}$ be the solution of the following SDE defined on $[t, T]$ :

$$
Y_{s}^{t, z}=-\int_{t}^{s} g(r, z) d r+z \cdot\left(W_{s}-W_{t}\right)
$$

From (40), we have

$$
\mathscr{E}_{g}\left[\lambda Y_{s}^{t, z} \mid \mathscr{F}_{r}\right] \geq \lambda \mathscr{E}_{g}\left[Y_{s}^{t, z} \mid \mathscr{F}_{r}\right]=\lambda Y_{r}^{t, z}, \quad t \leq r \leq s \leq T .
$$

Thus, $\left(\lambda Y_{s}^{t, z}\right)_{s \in[t, T]}$ is an $\mathscr{E}_{g}$-submartingale. From the decomposition theorem of $\mathscr{E}_{g}$-supermartingale (see [15]), it follows that there exists an increasing process $\left(A_{s}\right)_{s \in[t, T]}$ such that

$$
\lambda Y_{s}^{t, z}=-\int_{t}^{s} g\left(r, Z_{r}\right) d r+A_{s}-A_{t}+\int_{t}^{s} Z_{r} \cdot d W_{r}
$$

$$
s \in[t, T] .
$$

This with $\lambda Y_{s}^{t, z}=-\int_{t}^{s} \lambda g(r, z) \mathrm{d} r+\int_{t}^{s} \lambda z \cdot d W_{r}$ yields $Z_{r} \equiv \lambda z$ and

$$
\lambda g(r, z) \leq g(r, \lambda z), \quad d P \times d t \text { a.s. }
$$

At last, we prove that $g$ is positively homogeneous with respect to $z$. From (iii), we can obtain that for each fixed $\lambda>0$ and $\xi \in L^{2}\left(\Omega, \mathscr{F}_{T}, P\right)$,

$$
\frac{1}{\lambda} \mathscr{E}_{g}\left[\lambda \xi \mid \mathscr{F}_{t}\right] \leq \mathscr{E}_{g}\left[\xi \mid \mathscr{F}_{t}\right], \quad \text { a.s., } \forall t \in[0, T],
$$

that is,

$$
\mathscr{E}_{g}\left[\lambda \xi \mid \mathscr{F}_{t}\right] \leq \lambda \mathscr{E}_{g}\left[\xi \mid \mathscr{F}_{t}\right], \quad \text { a.s., } \forall t \in[0, T] .
$$

Thus, we have

$$
\mathscr{E}_{g}\left[\lambda \xi \mid \mathscr{F}_{t}\right]=\lambda \mathscr{E}_{g}\left[\xi \mid \mathscr{F}_{t}\right], \quad \text { a.s., } \forall t \in[0, T] .
$$

Obviously, if $\lambda=0$, (47) still holds. Thus, for each $\lambda \geq 0$,

$$
\mathscr{E}_{g}\left[\lambda \xi \mid \mathscr{F}_{t}\right]=\lambda \mathscr{E}_{g}\left[\xi \mid \mathscr{F}_{t}\right], \quad \text { a.s., } \forall t \in[0, T] .
$$

For each $(t, z) \in[0, T] \times R^{d}$, let $Y^{t, z}$ be the solution of SDE (34). From (48), for each $\lambda \geq 0$, we have

$$
\mathscr{E}_{g}\left[\lambda Y_{s}^{t, z} \mid \mathscr{F}_{r}\right]=\lambda \mathscr{E}_{g}\left[Y_{s}^{t, z} \mid \mathscr{F}_{r}\right]=\lambda Y_{r}^{t, z}, \quad t \leq r \leq s \leq T .
$$

This implies that there exists a process $Z^{t, z, \lambda}$ such that

$$
\lambda Y_{s}^{t, z}=-\int_{t}^{s} g\left(r, Z_{r}^{t, z, \lambda}\right) d r+\int_{t}^{s} Z_{r}^{t, z, \lambda} \cdot d W_{r}, \quad s \in[t, T] .
$$

Comparing this with $\lambda Y_{s}^{t, z}=-\int_{t}^{s} \lambda g(r, z) d r+\int_{t}^{s} \lambda z \cdot d W_{r}$, it follows that $Z_{r}^{t, z, \lambda} \equiv \lambda z$ and

$$
\lambda g(r, z)=g(r, \lambda z), \quad d P \times d t \text { a.s. }
$$

(iv) $\Rightarrow$ (iii): By comparison theorem (for example, we can see [3]), it is easy to obtain (iii).

(iii) $\Rightarrow($ i): Suppose (iii) holds. From (iii) and by Remark 8 (i), we have

$$
\begin{gathered}
\forall(X, k) \in \mathscr{L}\left(\Omega, \mathscr{F}_{T}, P\right) \times R, \\
\mathscr{E}_{g}\left[X+k \mid \mathscr{F}_{t}\right]=\mathscr{E}_{g}\left[X \mid \mathscr{F}_{t}\right]+k \quad \text { a.s., } \\
\forall(X, \lambda) \in \mathscr{L}\left(\Omega, \mathscr{F}_{T}, P\right) \times R, \\
\mathscr{E}_{g}\left[\lambda X \mid \mathscr{F}_{t}\right] \geq \lambda \mathscr{E}_{g}\left[X \mid \mathscr{F}_{t}\right] \quad \text { a.s. }
\end{gathered}
$$


From (53), we can deduce that for each bounded variable $\zeta \in$ $\mathscr{F}_{t}$,

$\forall X \in \mathscr{L}\left(\Omega, \mathscr{F}_{T}, P\right), \quad \mathscr{E}_{g}\left[\zeta X \mid \mathscr{F}_{t}\right] \geq \zeta \mathscr{E}_{g}\left[X \mid \mathscr{F}_{t}\right] \quad$ a.s.

In fact, let $\left\{A_{i}\right\}_{i=1}^{m}$ be a $\mathscr{F}_{t}$-measurable partition of $\Omega$ and let $\lambda_{i} \in R(i=1,2, \ldots, m)$. By (53), we have

$$
\begin{aligned}
\mathscr{E}_{g}\left[\sum_{i=1}^{m} \lambda_{i} 1_{A_{i}} X \mid \mathscr{F}_{t}\right] & =\sum_{i=1}^{m} 1_{A_{i}} \mathscr{E}_{g}\left[\lambda_{i} X \mid \mathscr{F}_{t}\right] \\
& \geq \sum_{i=1}^{m} 1_{A_{i}} \lambda_{i} \mathscr{E}_{g}\left[X \mid \mathscr{F}_{t}\right] \text { a.s. }
\end{aligned}
$$

In other words, for each $X \in \mathscr{L}\left(\Omega, \mathscr{F}_{T}, P\right)$ and each simple function $\zeta \in \mathscr{L}\left(\Omega, \mathscr{F}_{t}, P\right)$,

$$
\mathscr{E}_{g}\left[\zeta X \mid \mathscr{F}_{t}\right] \geq \zeta \mathscr{E}_{g}\left[X \mid \mathscr{F}_{t}\right] \quad \text { a.s. }
$$

Thus, thanks to Remark 8(ii), it follows that (54) is true.

The main idea of the following proof is derived from [7]. Given $\xi \in \mathscr{L}\left(\Omega, \mathscr{F}_{T}, P\right)$ and convex function $\varphi$ such that $\varphi(\xi) \in \mathscr{L}\left(\Omega, \mathscr{F}_{T}, P\right)$, we set $\eta_{t}=\varphi_{-}^{\prime}\left(\mathscr{E}_{g}\left[\xi \mid \mathscr{F}_{t}\right]\right)$. Then $\eta_{t}$ is $\mathscr{F}_{t}$-measurable. Since $\varphi$ is convex, we have

$$
\varphi(x)-\varphi(y) \geq \varphi_{-}^{\prime}(y)(x-y), \quad \forall x, y \in R .
$$

Take $x=\xi, y=\mathscr{E}_{g}\left[\xi \mid \mathscr{F}_{t}\right]$. Then we have

$$
\varphi(\xi)-\varphi\left(\mathscr{E}_{g}\left[\xi \mid \mathscr{F}_{t}\right]\right) \geq \eta_{t}\left(\xi-\mathscr{E}_{g}\left[\xi \mid \mathscr{F}_{t}\right]\right) \quad \text { a.s. }
$$

For each $n \in N$, we define

$$
\Omega_{t, n}:=\left\{\left|\mathscr{E}_{g}\left[\xi \mid \mathscr{F}_{t}\right]\right|+\left|\eta_{t}\right|+\left|\varphi\left(\mathscr{E}_{g}\left[\xi \mid \mathscr{F}_{t}\right]\right)\right| \leq n\right\},
$$

so we have

$$
\begin{gathered}
\mathscr{E}_{g}\left[1_{\Omega_{t, n}} \varphi(\xi) \mid \mathscr{F}_{t}\right] \\
\geq \mathscr{E}_{g}\left[1_{\Omega_{t, n}} \varphi\left(\mathscr{E}_{g}\left[\xi \mid \mathscr{F}_{t}\right]\right)-1_{\Omega_{t, n}} \eta_{t} \mathscr{E}_{g}\left[\xi \mid \mathscr{F}_{t}\right]\right. \\
\left.+1_{\Omega_{t, n}} \eta_{t} \xi \mid \mathscr{F}_{t}\right] \quad \text { a.s. }
\end{gathered}
$$

By the definition of $1_{\Omega_{t, n}}$, we know

$$
1_{\Omega_{t, n}} \varphi\left(\mathscr{E}_{g}\left[\xi \mid \mathscr{F}_{t}\right]\right)-1_{\Omega_{t, n}} \eta_{t} \mathscr{E}_{g}\left[\xi \mid \mathscr{F}_{t}\right] \in \mathscr{L}\left(\Omega, \mathscr{F}_{t}, P\right) .
$$

Thus, in view of (52) and from Proposition 10, we can get

$$
\begin{aligned}
\mathscr{E}_{g}\left[1_{\Omega_{t, n}} \varphi(\xi) \mid \mathscr{F}_{t}\right] \geq & 1_{\Omega_{t, n}} \varphi\left(\mathscr{E}_{g}\left[\xi \mid \mathscr{F}_{t}\right]\right) \\
& -1_{\Omega_{t, n}} \eta_{t} \mathscr{E}_{g}\left[\xi \mid \mathscr{F}_{t}\right] \\
& +\mathscr{E}_{g}\left[1_{\Omega_{t, n}} \eta_{t} \xi \mid \mathscr{F}_{t}\right] \text { a.s. }
\end{aligned}
$$

Moreover, from (54), considering that $1_{\Omega_{t, n}} \eta_{t} \in \mathscr{F}_{t}$ and is bounded by $n$, we can get

$$
\mathscr{E}_{g}\left[1_{\Omega_{t, n}} \eta_{t} \xi \mid \mathscr{F}_{t}\right] \geq 1_{\Omega_{t, n}} \eta_{t} \mathscr{E}_{g}\left[\xi \mid \mathscr{F}_{t}\right] \quad \text { a.s. }
$$

Hence, we can deduce that for each $n \in N$,

$$
\mathscr{E}_{g}\left[1_{\Omega_{t, n}} \varphi(\xi) \mid \mathscr{F}_{t}\right] \geq 1_{\Omega_{t, n}} \varphi\left(\mathscr{E}_{g}\left[\xi \mid \mathscr{F}_{t}\right]\right) \quad \text { a.s. }
$$

Finally, thanks to Remark 8 (ii) again, we can get

$$
\mathscr{E}_{g}\left[\varphi(\xi) \mid \mathscr{F}_{t}\right] \geq \varphi\left(\mathscr{E}_{g}\left[\xi \mid \mathscr{F}_{t}\right]\right) \text { a.s. }
$$

Hence, Jensen's inequality for $\mathscr{E}_{g}\left[\cdot \mid \mathscr{F}_{t}\right]$ holds in general. The proof of Theorem 13 is complete.

Example 14. Suppose $H$ is a bounded, convex, and closed subset of $R^{d}$ and $D=$ the set of $R^{d}$-valued continuous processes $\left(v_{t}\right)_{t \in[0, T]}$ such that for each $t, v_{t} \in H$ a.s.. Define the probability measure $Q^{v}$ by

$$
\frac{d Q^{v}}{d P}=\mathrm{e}^{-(1 / 2) \int_{0}^{T}\left|v_{s}\right|^{2} d s+\int_{0}^{T} v_{s} \cdot d W_{s}} .
$$

Thus, for any convex function $\varphi$,

$$
\begin{array}{r}
\varphi\left(\text { ess } \sup _{v \in D} E_{Q^{v}}\left[\xi \mid \mathscr{F}_{t}\right]\right) \leq \text { ess } \sup _{v \in D} E_{Q^{v}}\left[\varphi(\xi) \mid \mathscr{F}_{t}\right], \\
\text { a.s., } \forall t \in[0, T],
\end{array}
$$

whenever $\xi, \varphi(\xi) \in \mathscr{L}\left(\Omega, \mathscr{F}_{T}, P\right)$.

Proof. Let $g(t, z)=$ ess $\sup _{v \in D} v_{t} \cdot z$. Obviously, $g(t, z)$ is superhomogeneous and positively homogeneous with respect to $z$. and satisfies (A.1) and (A.2).

From El Karoui and Quenez [16], we have

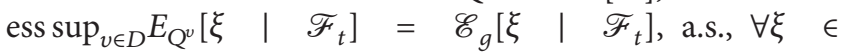
$L^{2}\left(\Omega, \mathscr{F}_{T}, P\right)$. Now we prove $\left.\operatorname{ess}^{\sup _{v \in D} E_{Q^{v}}[\xi \mid} \mathscr{F}_{t}\right]=$ $\mathscr{E}_{g}\left[\xi \mid \mathscr{F}_{t}\right]$, a.s., $\forall \xi \in \mathscr{L}\left(\Omega, \mathscr{F}_{T}, P\right)$. Indeed, for any $\xi \in \mathscr{L}\left(\Omega, \mathscr{F}_{T}, P\right)$, there exists $1<p<2$ such that $\xi \in L^{p}\left(\Omega, \mathscr{F}_{T}, P\right)$. Let $\xi^{n}=(\xi \wedge n) \vee(-n), n=1,2, \ldots$ Clearly, for each $n, \xi^{n} \in L^{2}\left(\Omega, \mathscr{F}_{T}, P\right)$, then

$$
\text { ess } \sup _{v \in D} E_{Q^{v}}\left[\xi^{n} \mid \mathscr{F}_{t}\right]=\mathscr{E}_{g}\left[\xi^{n} \mid \mathscr{F}_{t}\right] \text {, a.s. }
$$

Since

$$
\begin{aligned}
\text { ess } \sup _{v \in D} E_{Q^{v}}\left[\xi^{n} \mid \mathscr{F}_{t}\right] \\
\quad=\operatorname{ess} \sup _{v \in D}\left(E_{Q^{v}}\left[\xi^{n}-\xi \mid \mathscr{F}_{t}\right]+E_{Q^{v}}\left[\xi \mid \mathscr{F}_{t}\right]\right) \\
\quad \leq \operatorname{ess} \sup _{v \in D} E_{Q^{v}}\left[\xi^{n}-\xi \mid \mathscr{F}_{t}\right]+\operatorname{ess} \sup _{v \in D} E_{Q^{v}}\left[\xi \mid \mathscr{F}_{t}\right],
\end{aligned}
$$

we have

$$
\begin{gathered}
\mathscr{E}_{g}\left[\xi^{n} \mid \mathscr{F}_{t}\right]-\operatorname{ess} \sup _{v \in D} E_{Q^{v}}\left[\xi \mid \mathscr{F}_{t}\right] \\
\leq \operatorname{ess} \sup _{v \in D} E_{Q^{v}}\left[\xi^{n}-\xi \mid \mathscr{F}_{t}\right] .
\end{gathered}
$$

With an approach similar to the one above, we can get easily that

$$
\begin{aligned}
\mathscr{E}_{g}\left[\xi^{n} \mid \mathscr{F}_{t}\right] & -\operatorname{ess} \sup _{v \in D} E_{Q^{v}}\left[\xi \mid \mathscr{F}_{t}\right] \\
& \geq \operatorname{ess} \inf _{v \in D} E_{Q^{v}}\left[\xi^{n}-\xi \mid \mathscr{F}_{t}\right] .
\end{aligned}
$$


Combining (42) with (43), we have

$$
\begin{gathered}
\left|\mathscr{E}_{g}\left[\xi^{n} \mid \mathscr{F}_{t}\right]-\operatorname{ess} \sup _{v \in D} E_{Q^{v}}\left[\xi \mid \mathscr{F}_{t}\right]\right| \\
\leq\left(\left|\operatorname{ess} \inf _{v \in D} E_{Q^{v}}\left[\xi^{n}-\xi \mid \mathscr{F}_{t}\right]\right|\right. \\
\left.\vee\left|\operatorname{ess} \sup _{v \in D} E_{Q^{v}}\left[\xi^{n}-\xi \mid \mathscr{F}_{t}\right]\right|\right) \\
\leq \operatorname{ess} \sup _{v \in D} E_{Q^{v}}\left[\left|\xi^{n}-\xi\right| \mid \mathscr{F}_{t}\right] .
\end{gathered}
$$

By Hölder's inequality and noting

that $\left(\mathrm{e}^{-(1 / 2) \int_{0}^{t}\left|v_{s}\right|^{2} d s+\int_{0}^{t} v_{s} \cdot d W_{s}}\right)_{t \in[0, T]} \quad$ and $\left(\mathrm{e}^{-(1 / 2) \int_{0}^{t}\left|q v_{s}\right|^{2} d s+\int_{0}^{t} q v_{s} \cdot d W_{s}}\right)_{t \in[0, T]}$ are both martingales with respect to $\left(\mathscr{F}_{t}\right)_{t \in[0, T]}$, we can obtain

$$
\begin{aligned}
E_{Q^{v}}\left[\left|\xi^{n}-\xi\right| \mid \mathscr{F}_{t}\right] \\
\quad=\frac{E\left[\left|\xi^{n}-\xi\right|\left(d Q^{v} / d P\right) \mid \mathscr{F}_{t}\right]}{E\left[\left(d Q^{v} / d P\right) \mid \mathscr{F}_{t}\right]} \\
\quad \leq \frac{\left(E\left[\left|\xi^{n}-\xi\right|^{p} \mid \mathscr{F}_{t}\right]\right)^{1 / p}\left(E\left[\left(d Q^{v} / d P\right)^{q} \mid \mathscr{F}_{t}\right]\right)^{1 / q}}{E\left[\left(d Q^{v} / d P\right) \mid \mathscr{F}_{t}\right]} \\
\leq \mathrm{e}^{(1 / 2)(q-1) \mu^{2} T}\left(E\left[\left|\xi^{n}-\xi\right|^{p} \mid \mathscr{F}_{t}\right]\right)^{1 / p},
\end{aligned}
$$

where $(1 / p)+(1 / q)=1$. It then follows from Lebesgue's dominated convergence theorem that

$$
\text { ess } \sup _{v \in D} E_{Q^{v}}\left[\left|\xi^{n}-\xi\right| \mid \mathscr{F}_{t}\right] \longrightarrow 0, \quad \text { as } n \longrightarrow \infty \text {. }
$$

Hence,

$$
\left|\mathscr{E}_{g}\left[\xi^{n} \mid \mathscr{F}_{t}\right]-\operatorname{ess} \sup _{v \in D} E_{Q^{v}}\left[\xi \mid \mathscr{F}_{t}\right]\right| \longrightarrow 0, \quad \text { as } n \longrightarrow \infty .
$$

On the other hand, from Remark 8(i), we have

$$
\mathscr{E}_{g}\left[\xi^{n} \mid \mathscr{F}_{t}\right] \longrightarrow \mathscr{E}_{g}\left[\xi \mid \mathscr{F}_{t}\right], \quad \text { as } n \longrightarrow \infty
$$

Thus,

$$
\begin{array}{r}
\mathscr{E}_{g}\left[\xi \mid \mathscr{F}_{t}\right]=\operatorname{ess} \sup _{v \in D} E_{Q^{v}}\left[\xi \mid \mathscr{F}_{t}\right], \\
\text { a.s., } \forall \xi \in \mathscr{L}\left(\Omega, \mathscr{F}_{T}, P\right) .
\end{array}
$$

Applying Theorem 13, we have

$$
\varphi\left(\text { ess } \sup _{v \in D} E_{Q^{v}}\left[\xi \mid \mathscr{F}_{t}\right]\right) \leq \text { ess } \sup _{v \in D} E_{Q^{v}}\left[\varphi(\xi) \mid \mathscr{F}_{t}\right], \quad \text { a.s. }
$$

Definition 15. Suppose $g$ satisfies (A.1) and (A.2). A process $\left(X_{t}\right)_{t \in[0, T]}$ satisfying that for each $t, X_{t} \in \mathscr{L}\left(\Omega, \mathscr{F}_{t}, P\right)$ is called a generalized Peng's $g$-martingale (resp., generalized Peng's $g$-supermartingale, generalized Peng's $g$ submartingale), if for any $t, s$ satisfying $t \leq s \leq T$,

$$
\mathscr{E}_{g}\left[X_{s} \mid \mathscr{F}_{t}\right]=X_{t} \quad\left(\text { resp. } \leq X_{t}, \geq X_{t}\right) \text {, a.s. }
$$

Applying Theorem 13, immediately we have the following.

Theorem 16. Suppose $g$ is independent of $y$, superhomogeneous and positively homogeneous with respect to $z$ and satisfies (A.1) and (A.2). If $\left(X_{t}\right)_{t \in[0, T]}$ is a generalized Peng's $g$-martingale and $\varphi$ is a convex function such that $\varphi\left(X_{t}\right) \in$ $\mathscr{L}\left(\Omega, \mathscr{F}_{t}, P\right)$, then $\left(\varphi\left(X_{t}\right)\right)_{t \in[0, T]}$ is a generalized Peng's $g$ submartingale.

Remark 17. Suppose $g$ is independent of $y$, superhomogeneous and positively homogeneous with respect to $z$ and satisfies (A.1) and (A.2). Similarly, we can get the following.

(i) If $\left(X_{t}\right)_{t \in[0, T]}$ is a generalized Peng's $g$-submartingale and $\varphi$ is a convex and increasing function such that $\varphi\left(X_{t}\right) \in \mathscr{L}\left(\Omega, \mathscr{F}_{t}, P\right)$, then $\left(\varphi\left(X_{t}\right)\right)_{t \in[0, T]}$ is a generalized Peng's $g$-submartingale.

(ii) If $\left(X_{t}\right)_{t \in[0, T]}$ is a generalized Peng's $g$-supermartingale and $\varphi$ is a convex and decreasing function such that $\varphi\left(X_{t}\right) \in \mathscr{L}\left(\Omega, \mathscr{F}_{t}, P\right)$, then $\left(\varphi\left(X_{t}\right)\right)_{t \in[0, T]}$ is a generalized Peng's $g$-submartingale.

Example 18. (i) Let $g=\mu|z|$ and $\varphi(x)=(x-a)^{+}$where $a \in R$. Obviously, $g$ satisfies the assumptions of Remark 17 and $\varphi$ is a convex and increasing function. By Remark 17 (i), we have the following: suppose $\left(X_{t}\right)_{t \in[0, T]}$ is a $\mathscr{E}^{\mu}$-submartingale, then $\left(\left(X_{t}-a\right)^{+}\right)_{t \in[0, T]}$ is a $\mathscr{E}^{\mu}$-submartingale.

(ii) Let $g=\mu|z|$ and $\varphi(x)=(x-b)^{-}$where $b \in R$. With the similar argument, we have the following: suppose $\left(Y_{t}\right)_{t \in[0, T]}$ is a $\mathscr{E}^{\mu}$-supermartingale, then $\left(\left(Y_{t}-b\right)^{-}\right)_{t \in[0, T]}$ is a $\mathscr{E}^{\mu}$-submartingale.

\section{Acknowledgments}

The author would like to thank the anonymous referees for their careful reading of this paper and valuable suggestions. The author thanks the partial support from the National Natural Science Foundation of China (Grant no. 11171179), the Doctoral Program Foundation of Ministry of Education of China (Grant no. 20093705110002 and 20123705120005), and the Natural Science Foundation of Shandong Province of China (Grant no. ZR2012AQ009).

\section{References}

[1] E. Pardoux and S. Peng, "Adapted solution of a backward stochastic differential equation," Systems \& Control Letters, vol. 14, no. 1, pp. 55-61, 1990.

[2] S. Peng, "A generalized dynamic programming principle and Hamilton-Jacobi-Bellman equation," Stochastics and Stochastics Reports, vol. 38, no. 2, pp. 119-134, 1992. 
[3] S. Peng, "Backward SDE and related g-expectation," in Backward Stochastic Differential Equations, N. El Karoui and L. Mazliak, Eds., vol. 364 of Pitman Research Notes in Mathematics Series, pp. 141-159, Longman, Harlow, UK, 1997.

[4] Ph. Briand, F. Coquet, Y. Hu, J. Mémin, and S. Peng, "A converse comparison theorem for BSDEs and related properties of $g$ expectation," Electronic Communications in Probability, vol. 5, pp. 101-117, 2000.

[5] Z. Chen, R. Kulperger, and L. Jiang, “Jensen's inequality for gexpectation. I," Comptes Rendus Mathématique, vol. 337, no. 11, pp. 725-730, 2003.

[6] Z. Chen, R. Kulperger, and L. Jiang, “Jensen's inequality for gexpectation. II," Comptes Rendus Mathématique, vol. 337, no. 12, pp. 797-800, 2003.

[7] L. Jiang, "Representation theorems for generators of backward stochastic differential equations and their applications," Stochastic Processes and their Applications, vol. 115, no. 12, pp. 1883-1903, 2005.

[8] Y. Hu, "On Jensen's inequality for $g$-expectation and for nonlinear expectation," Archiv der Mathematik, vol. 85, no. 6, pp. 572-680, 2005.

[9] L. Jiang, "Jensen's inequality for backward stochastic differential equations," Chinese Annals of Mathematics Series B, vol. 27, no. 5, pp. 553-564, 2006.

[10] Ph. Briand, B. Delyon, Y. Hu, E. Pardoux, and L. Stoica, " $L^{p}$ solutions of backward stochastic differential equations," Stochastic Processes and their Applications, vol. 108, no. 1, pp. 109-129, 2003.

[11] F. Hu and Z. Chen, "Generalized Peng's g-expectations and related properties," Statistics \& Probability Letters, vol. 80, no. 3-4, pp. 191-195, 2010.

[12] Z. Chen and R. Kulperger, "Minimax pricing and Choquet pricing," Insurance: Mathematics and Economics, vol. 38, no. 3, pp. 518-528, 2006.

[13] F. Coquet, Y. Hu, J. Mémin, and S. Peng, "Filtration-consistent nonlinear expectations and related g-expectations," Probability Theory and Related Fields, vol. 123, no. 1, pp. 1-27, 2002.

[14] S. Peng, "Modelling derivatives pricing mechanisms with their generating functions," http://arxiv.org/abs/math/0605599, 2006.

[15] S. Peng, "Monotonic limit theorem of BSDE and nonlinear decomposition theorem of Doob-Meyer's type," Probability Theory and Related Fields, vol. 113, no. 4, pp. 473-499, 1999.

[16] N. El Karoui and M.-C. Quenez, "Dynamic programming and pricing of contingent claims in an incomplete market," SIAM Journal on Control and Optimization, vol. 33, no. 1, pp. 29-66, 1995. 


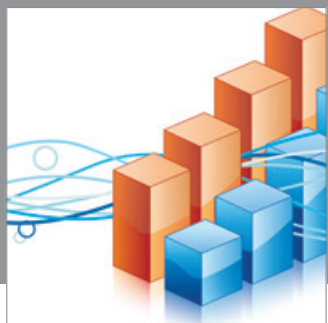

Advances in

Operations Research

mansans

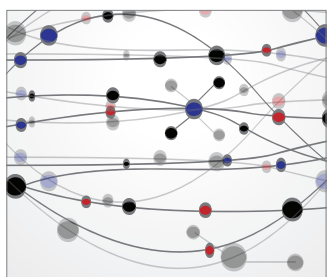

The Scientific World Journal
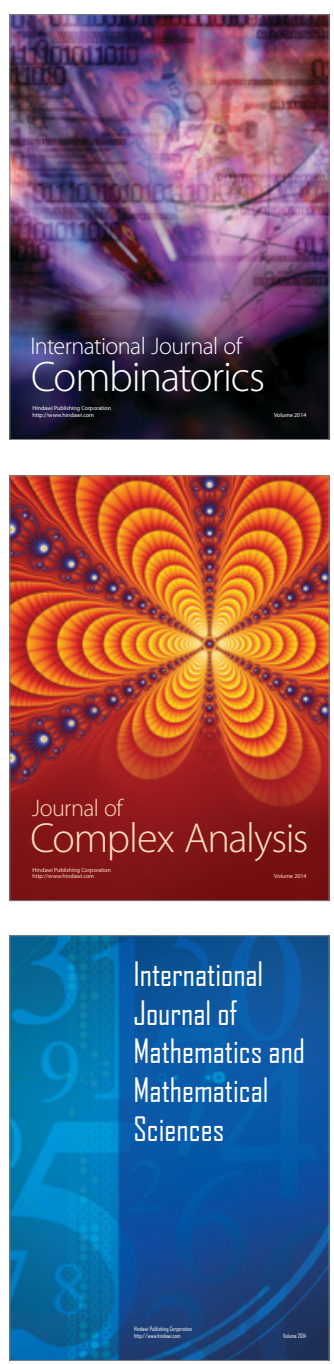
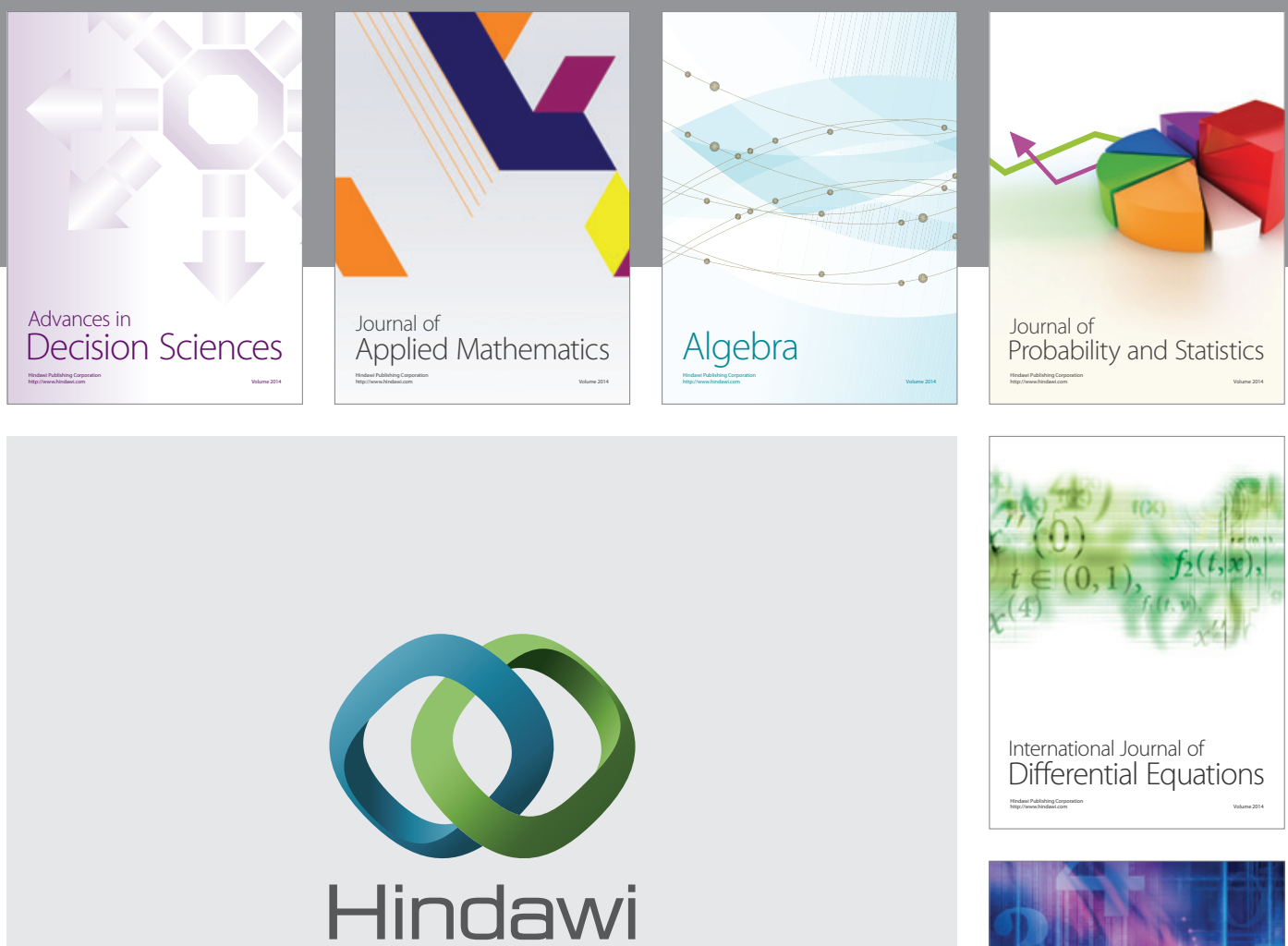

Submit your manuscripts at http://www.hindawi.com
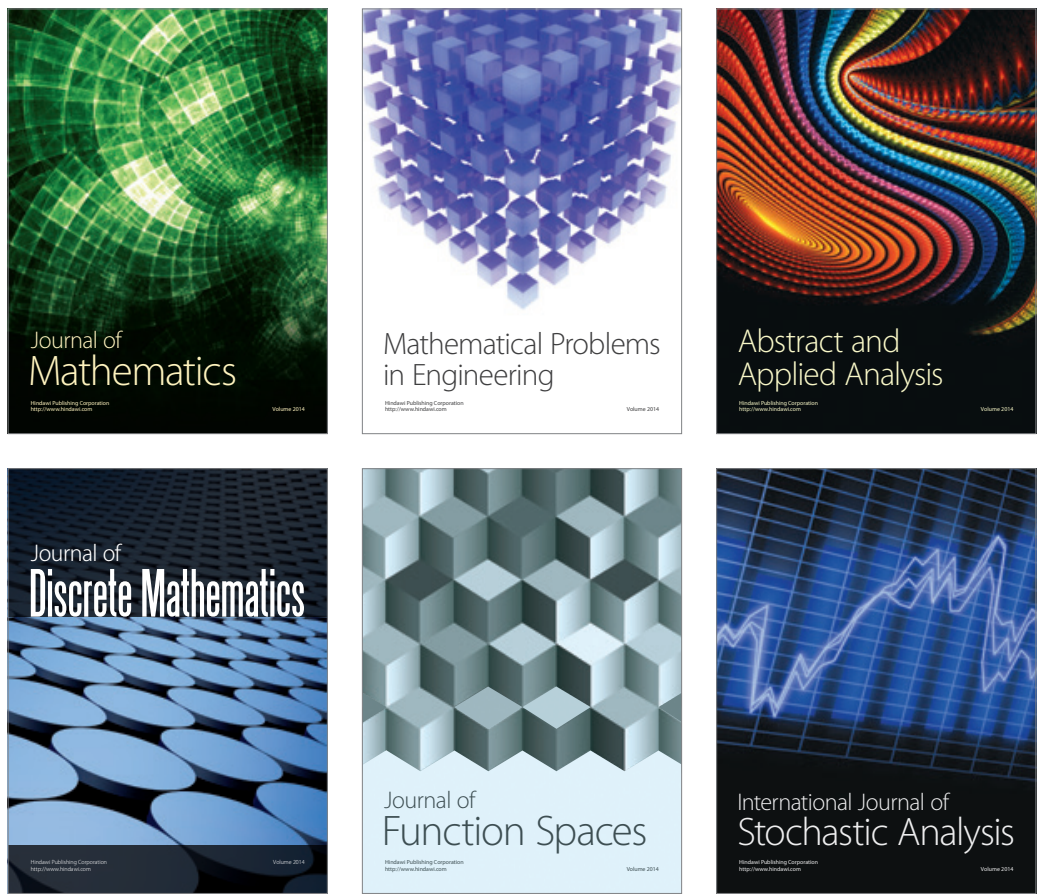

Journal of

Function Spaces

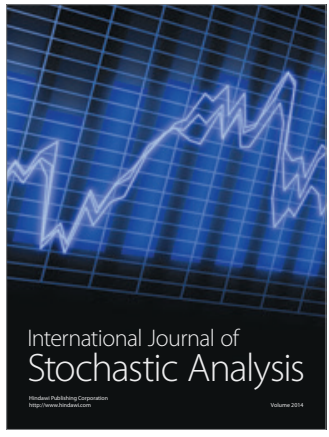

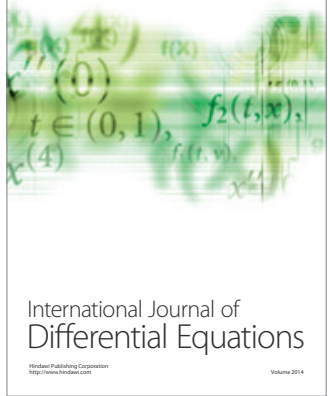
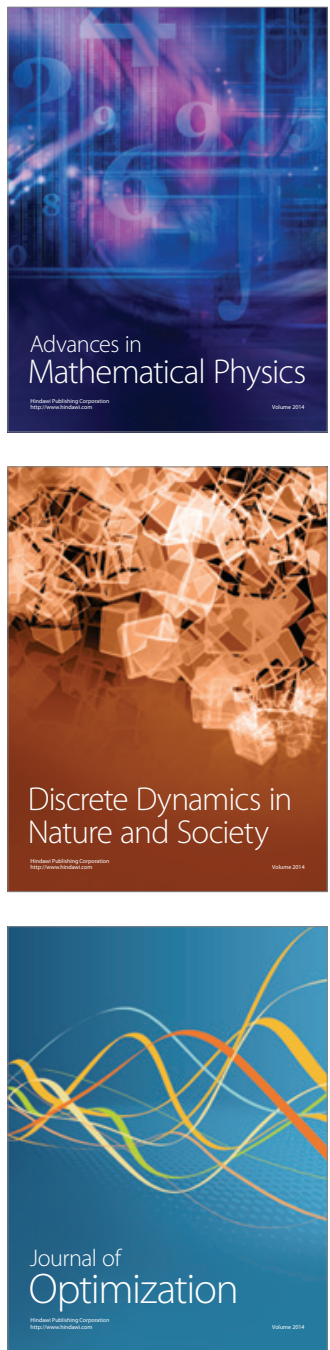